\title{
An industrial case study: QbD to accelerate time-to-market of a drug product
}

\author{
Madalena Testas ${ }^{1}$, Tiago da Cunha Sais², Leonardo Piccoli Medinilha² , Katia Nami Ito Niwa², \\ Lucas Sponton de Carvalho², Silvia Duarte Maia², Anderson Flores³ , Lígia Pedroso Braz', \\ José Cardoso Menezes ${ }^{1 *}$ and Cássio Yooiti Yamakawa ${ }^{2,4}$
}

\begin{abstract}
The use of a Quality by Design (QbD) approach in the development of pharmaceutical products is known to bring many advantages to the table, such as increased product and process knowledge, robust manufacturing processes, and regulatory flexibility regarding changes during the commercial phase. However, many companies still adhere to a more traditional pharmaceutical process development-in some cases due to the difficulty of going from a theoretical view of QbD to its actual application. This article presents a real-world case study for the development of an industrial pharmaceutical drug product (oral solid dosage form) using the QbD methodology, demonstrating the activities involved and the gains in obtaining systematic process and product knowledge.
\end{abstract}

Keywords: Pharmaceutical product development, Quality by design, Knowledge management, Quality risk management, Design of experiments, Pharmaceutical scale-up, Control strategy, Product lifecycle management

\section{Introduction}

In 1992, Dr. Joseph M. Juran introduced the concept of quality being designed into a product and that most quality issues were related to the way in which the product was designed in the first place (Yu et al., 2014). Over time, this Quality by Design (QbD) approach was translated into the pharmaceutical industry, reaching its most important evolution steps with the publication of three guidelines by the International Council for Harmonisation of Technical Requirements for Pharmaceuticals for Human Use (ICH), namely, ICH Q8(R2), Q9, and Q10 (ICH, 2009; ICH, 2005; ICH, 2008). These guidelines describe the elements of $\mathrm{QbD}$ : pharmaceutical development, quality risk management, and pharmaceutical quality system.

ICH Q8(R2) defines QbD as "a systematic approach to development that begins with predefined objectives

\footnotetext{
*Correspondence: hello@4TuneEngineering.com

1 4Tune Engineering Lda., Av. António Augusto Aguiar 108-4,

1050-019 Lisbon, Portugal

Full list of author information is available at the end of the article
}

and emphasizes product and process understanding and process control, based on sound science and quality risk management." This is a clear and easy to understand description, at least in theory ( $\mathrm{ICH}, 2009)$. In its form, QbD can be explained as an orderly, well-planned procedure to assemble and deliver quality. For that, it is required an extensive comprehension of how the product and process factors impact quality (Malik et al., 2019).

But how to go from definitions and guidelines to an actual process and product development in a real-world situation? The uncertainty in the answer drives many companies away from $\mathrm{QbD}$ and to adhere to a more traditional approach to pharmaceutical development. Figure 1 represents a workflow with all the important elements that must be present in a $\mathrm{QbD}$ development of a pharmaceutical product.

Just like the ICH Q8(R2) guideline indicates, one of the first elements to be defined is the Quality Target Product Profile (QTPP) - a summary of the desirable quality characteristics a product should have to ensure the desired quality, taking into account safety and efficacy of the drug product to the patient (Yu et al., 2014; 


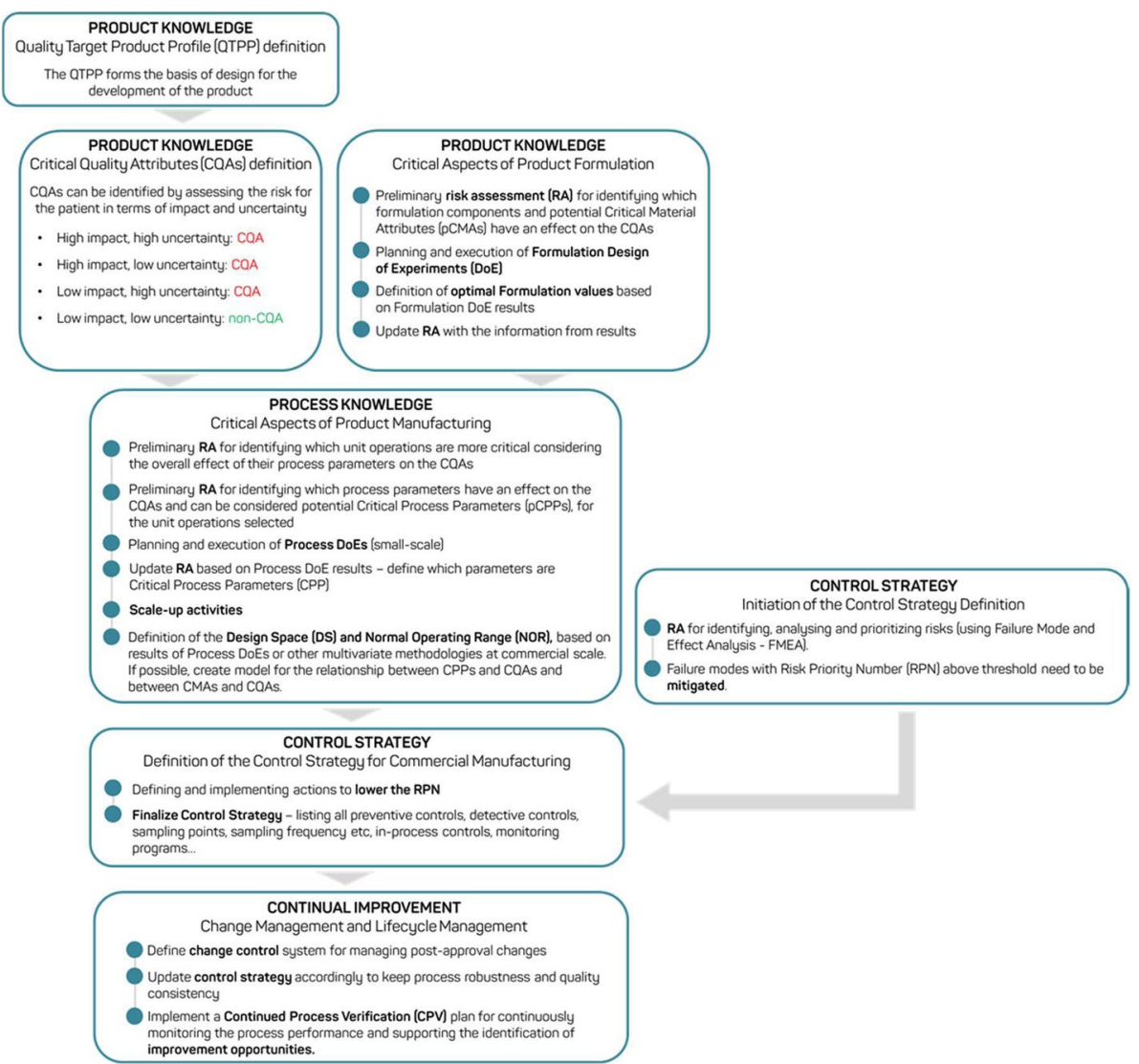

Fig. 1 Quality by Design methodology applied for a pharmaceutical product development

ISPE, 2011). The end goal of process development is the definition of a control strategy that ensures that the process consistently delivers a product with the quality for which it was designed. The multidimensional combination and interaction of process inputs that have demonstrated to maintain the Critical Quality Attributes (CQA, i.e., the product quality characteristics that are critical for ensuring the safety and efficacy from a patient's perspective) within their specification (and thus, assure quality) is called the Design Space (DS) (Yu et al., 2014; ICH, 2009; ISPE, 2011). This concept brings certain regulatory flexibility to the table, since alterations made within the DS are not considered changes (ICH, 2009). The elements represented in Fig. 1 are obtained using risk management and knowledge management methodologies. The combination of risk assessment (RA) and data analysis is one of the stone pillars for $\mathrm{QbD}$ and the opportunities for acquiring and managing knowledge based on this arrangement are central for a successful QbD pharmaceutical development and lifecycle management.

\section{A drug product development case study}

Herein, we describe how the QbD approach and its concepts, summarized in Fig. 1, were applied to a real-case development of a generic pharmaceutical drug product (DP), i.e., of a drug intended to be submitted to the regulatory agencies as an alternative to a brand-name drug (patent-protected). The project's goal was to develop a generic two-API (active pharmaceutical ingredient) solid dosage oral form using the QbD approach outlined in Fig.1, in order to obtain a deeper product and process understanding to expedite time to market, assure process assertiveness and reduce risk of defects after product launch. Limitations of this work are the ones typical of the development of a generic DP, where the physicochemical characteristics of the reference listed drug (patent-protected brand-name drug) must be considered. The proposed generic DP must be comparable to the innovator DP in dosage form, strength, route of administration, quality, performance characteristics, and intended use. So, the generic manufacturer must scientifically demonstrate that his product performs in the same way as the innovator drug with respect to pharmacokinetic and 
pharmacodynamic properties (e.g., by performing bioequivalence studies) for it to be approved for sale after the patent protections expire. This work is the result of a collaborative project between 4Tune Engineering and Libbs Farmacêutica.

\section{Materials and methods}

The pharmaceutical product considered in this case study consists of a generic two-API oral solid dosage form-coated tablets. Tablets are amongst the most common oral solid dosage forms and consist of a compressed powder formulation comprised of API(s) (or drug substance(s)) and inactive ingredients or excipients (e.g., fillers, binders, lubricants, disintegrants, coatings). A generic drug contains the same API as the original (patent-protected) innovator drug, but may vary in certain characteristics, such as the manufacturing process, formulation, and packaging. In this case study, the tablets have distinct dosages of the two active ingredients: one API is at very low amounts $(2.5 \mathrm{mg})$, whereas the other is at a very high dose (up to 200 to 400 times higher). The reference DP is already available in the market to patients, with no reported risks related to drug-drug compatibility or with safety concerns to the patients. The unit operations involved are those typical of the manufacturing process of a coated tablet form, such as materials dispensing, fluid bed granulation and drying, blending, compression, and tablet coating. For confidentiality reasons, the names of raw materials, intermediates and DP, manufacturing operations, parameter ranges, and other manufacturing details are not disclosed throughout this article. The results presented serve only the purpose of exemplifying the methodology used.

The authors' goal with this manuscript is to provide, in the form of a case study, a brief outline of the steps involved in the application of the QbD methodology in the development of a pharmaceutical product. It is out of this paper's scope to give a technical review or discussion of the methodologies and techniques comprised in the QbD toolkit, such as design of experiments and modelling approaches, and quality risk management tools. The interested reader should consult specialized literature for further methods' details. Methodology aspects related with design of experiments, multivariate analysis, modelling, and quality risk management are given, as required for the purpose of this work, along the " 5 " section, while going through the case study.

The designed experiments and analyses described herein were performed in software $\mathrm{JMP}^{\circledR}$ version 13 (SAS Institute Inc., Cary, NC, USA, 1989-2019). Principal Component Analysis (PCA) modelling and computer simulations were performed in MATLAB ${ }^{\circledR}$ version 2018a (The Math Works, Inc., Natick, MA, USA) and using
PLS_Toolbox version 8.7 (Eigenvector Research, Inc., Manson, WA, USA).

The QbD methodology followed along this project for knowledge and risk management was supported by the use of the iRISK ${ }^{\mathrm{TM}}$ platform (version 2.8) (iRISK, 2021) by the interacting multidisciplinary technical team. Several iRISK $^{\mathrm{TM}}$ tools were employed, such as Process Mapping, Critical Quality Attributes assessment tool, Cause-Effect matrix for risk assessment and criticality analysis, and Failure Mode and Effect Analysis (FMEA) for process risk assessment.

\section{Results and discussion}

How to combine risk and knowledge in pharmaceutical development

Following the QbD methodology (Fig. 1), one of the first activities conducted in this work was a criticality assessment (CA) for the identification of CQAs. For this, the project team gathered as much product-related information as possible from literature, specific data of the reference product, and the QTPP. Having a list with the product quality attributes and their respective target values/ranges is standard: fulfilling these targets is mandatory for batch release. However, assessing these characteristics from a risk-to-patient perspective might be more complex. From a list of about 20 potential Critical Quality Attributes (pCQAs) collected by the team, a ranking system for PCQAs' CA was applied based on a criticality score that considered the risk for the patient of each quality attribute. Specifically, the criticality score is a quantitative measure given by the product between uncertainty and impact. The uncertainty measures the relevance of the available information (e.g, literature, prior knowledge, in vitro, clinical data), i.e., if there is variation in a quality attribute, are the consequences for the patient well-known? The impact measures how severe will the change of a given quality attribute be in terms of efficacy, safety, and pharmacokinetics and pharmacodynamics. By setting up a criticality threshold and a numeric ranking, it is possible to have the quantification of risk and a more exact approach for defining the criticality. For the CA, the team employed a scoring scale with 5 levels (Impact score: 2 (none), 4 (low), 12 (moderate), 16 (high), and 20 (very high); Uncertainty score: 1 (very low), 2 (low), 3 (moderate), 5 (high), and 7 (very high)). During this exercise, the attributes with low uncertainty and low impact were not considered critical and, therefore, were classified as non-CQAs (Fig. 1); Quality attributes with low severity but high uncertainty were considered critical - unless more information had become available to lower their uncertainty. The use of a systematic quality risk management platform for this exercise, specifically iRISK $^{\mathrm{TM}}$ CQA Assessment tool (iRISK, 2021), ensured 
standardization of the definition of critical quality by allowing an alignment of methodologies, concepts and evaluation criteria by the involved technical teams. At the end of this step, the project team identified about fifteen CQAs, such as assay, content uniformity and dissolution of each API, water content, and impurities.

With a clear definition of the critical quality elements and respective targets, the manufacturing process can now be designed to meet those requirements (Fig.1). Five different manufacturing processes were then considered and evaluated by the technical team based on process knowledge and experience, and given the product's specificities (namely the technical challenges related with the manufacture of a DP having two APIs at extremely different concentrations). Figure 2 shows the process flowchart for the chosen process comprising 10-unit operations, including materials dispensing, powdered material seiving, solution/suspension preparation steps, fluid bed granulation and drying, blending, compression, and tablet coating.

In the next step of the QbD methodology (Fig. 1), the critical aspects of the product formulation and manufacturing process were assessed by following a combination of risk-based and data-driven approaches. A preliminary CA based on the reference product and/or similar products information (literature and prior knowledge) helped to identify which excipient and/or combination of excipients might present the highest risk of affecting the final product's quality. This CA was performed using the risk tool Cause-Effect Matrix (CEM) (iRISK, 2021). In general terms, a CEM involves rating process inputs to process outputs based on their interaction impact, and then ranking process inputs based on the order of importance of the process output to the customer (ISPE, 2017; ISPE/ PDA, 2019). For confidentiality reasons, the CEM generated at this stage of the project is not shown. It is similar to the CEM given in Fig. 4, but has the formulation excipients in rows. The risk of a formulation component affecting a given final product's CQA (entry of the CEM) was classified as low (score of 1), medium (score of 3), and high (score of 9) based on literature and prior knowledge, as stated above. This preliminary risk rank filtering of formulation components identified the two APIs and five excipients as having the highest impact on the product's quality. Then, a design of experiments (DoE) approach (Montgomery, 2020) was followed to characterize these formulation components' impact on the product's CQAs, and their respective interactions, and therefore define the optimal quantities of each excipient in the drug formulation. A designed experiment consists of a set of trials, in which multiple input factors (independent variables) are manipulated to determine their effect on one or more response variables (dependent variables); these trails are run at different factor values (known as levels). DoE provides an efficient framework to do experimentation and thus increase process and product understanding

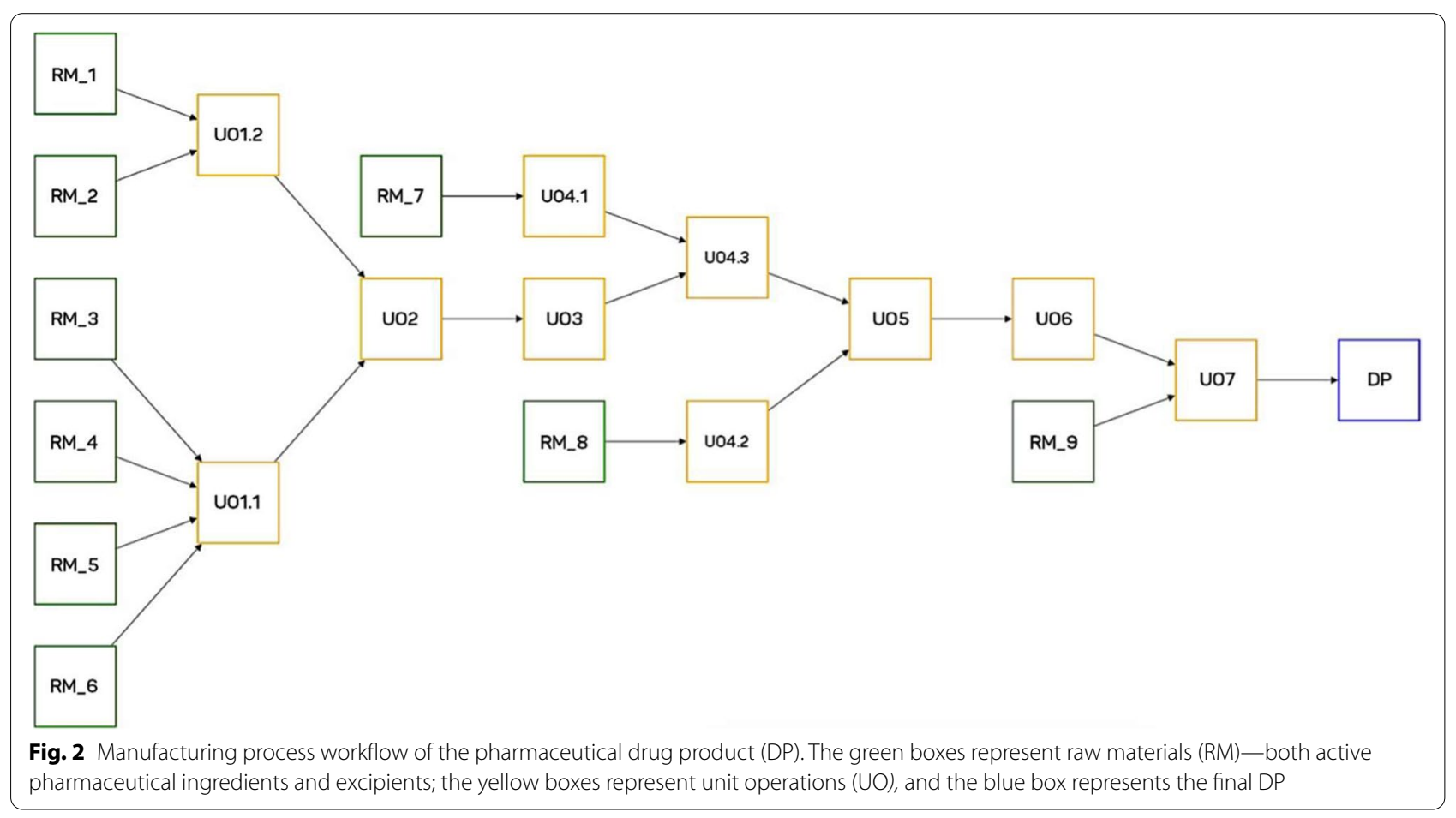


and optimize processes. In fact, DoE can be applied for different investigation objectives, such as (1) screening studies (where the goal is to discover which are the most important factors that affect the process under study, given a large set of potential factors), (2) optimization studies (involve determining optimal factor settings to achieve a desired process objective), (3) regression modelling (where is goal is to produce a detailed mathematical model quantifying the dependence of response variables on process inputs, instead of just examining how factors contribute to a response), and (4) robustness studies (involve determining operational settings that are least affected by noise factors or uncontrolled factors variations (e.g., environmental variation, manufacturing variation) that might be expected during the process to ensure that the process is robust to them).

In this case, the formulation DoE was created using as factors the ratio between APIs (API-1/API-2, where API-1 is the low dosage API and API-2 is the high dosage one) and the percentage of five excipients (selected in the previous $\mathrm{CA}$, as abovementioned). Based on the outcomes of the CA of the formulation components, the considered responses were the decrease in assay of each API, the amount of total impurities, and the amount of individual impurities of the final product. The type of screening design applied was a Definitive Screening Design (DSD) (SAS Institute, 2019). DSDs consist of an innovative and efficient class of screening designs, offering several advantages over standard screening designs (such as fractional factorial design). DSDs avoid confounding of effects (i.e., main effects are not confounded with each other or with two-way interactions) and can identify factors causing a nonlinear effect on the response (by employing three levels for each continuous factorlow, middle, and high - these designs allow estimation of quadratic model terms for continuous factors). Besides, DSDs require a small number of trials (e.g., with six or more factors, the minimum number of required runs is usually only a few more than twofold the number of factors). DSDs are appropriate for early-stage experimentation work, usually with four or more factors, and allow to perform screening, optimization, and robustness studies. These advantages of DSDs justified the selection of this type of screening against standard screening designs, such as fractional factorial designs, to perform the formulation screening and optimization studies, as a tradeoff between budgetary constraints (time and resources) and knowledge expected to extract from the experiments. By applying a DSD, the formulation DoE therefore consisted of 13 trials, and each factor assumed three levels (low, middle, high).

Based on the DoE outcomes, multivariate linear regression models were built describing the relationship between the formulation components and the responses evaluated. These models were then used for formulation optimization (SAS Institute, 2019), i.e., to estimate the amount of each formulation component required to minimize the impurity profile of the drug product and minimize the decrease in assay. The formulation optimization was performed on the reduced models, i.e., models constructed after removing non-significant terms from the initial full DoE models (terms with a $p$-value above 0.05 ). The following three factors remained in the optimized multivariate linear models: API-1/API-2 ratio, amount of stabilizer, and amount of Excipient A. The optimal settings for the formulation components are represented in red in Fig. 3 (red dotted lines and red values). Each plot shows the effect of a given factor ( $x$-axis) on each of the responses ( $y$-axis). For example, the profiles indicate that: a) the $\%$ of Stabilizer in the formulation affects all the five responses (assay and impurity levels) and a lower content of Stabilizer has a detrimental effect on the DP assay; b) the amount of Excipient $\mathrm{A}$ in the formulation has no impact on Unknown Impurity B (flat line) but affects the other impurities; c) while the ratio of APIs has no effect on the DP assay (horizontal line), lower values of API-1/ API-2 contribute to higher impurity levels of Unknown Impurity $\mathrm{A}$ and $\mathrm{B}$.

Next, a similar approach based on a CA exercise using the CEM risk tool (iRISK, 2021) was applied for defining the critical aspects of the manufacturing process, specifically to determine the Critical Process Parameters (CPPs)-Fig. 1. As per ICH Q8(R2), a CPP is "a process parameter whose variability has an impact on a CQA and therefore should be monitored or controlled to ensure the process produces the desired quality" (ICH, 2009).

The first step involved applying the CEM tool to rate the unit operations in terms of their impact on the product's CQAs (scoring scale: low $=1$; medium $=3$; high $=9$ ). These results supported a prioritization, in which the unit operations having the potential strongest impact (highest overall score) on the product's CQAs were assessed first. As shown in Fig. 4, unit operations UO2 and UO6 were the top-ranking ones.

These two process steps (UO2 and UO6) were then investigated by running DoEs with the goal of understanding which process parameters (PPs) were influencing the CQAs and in which extent. First, a prioritization step using the CEM tool (iRISK, 2021) was done in order to select from the original 20 PPs of UO2 and UO6, those to be considered for the DoEs. This PPs ranking was based on their level of impact in the final product's quality (scoring scale: low $=1$; medium $=3$; high $=9$ ), leading to the selection of 8 potential CPPs (pCPPs) for UO2, and 6 pCPPs for UO6 (not disclosed 


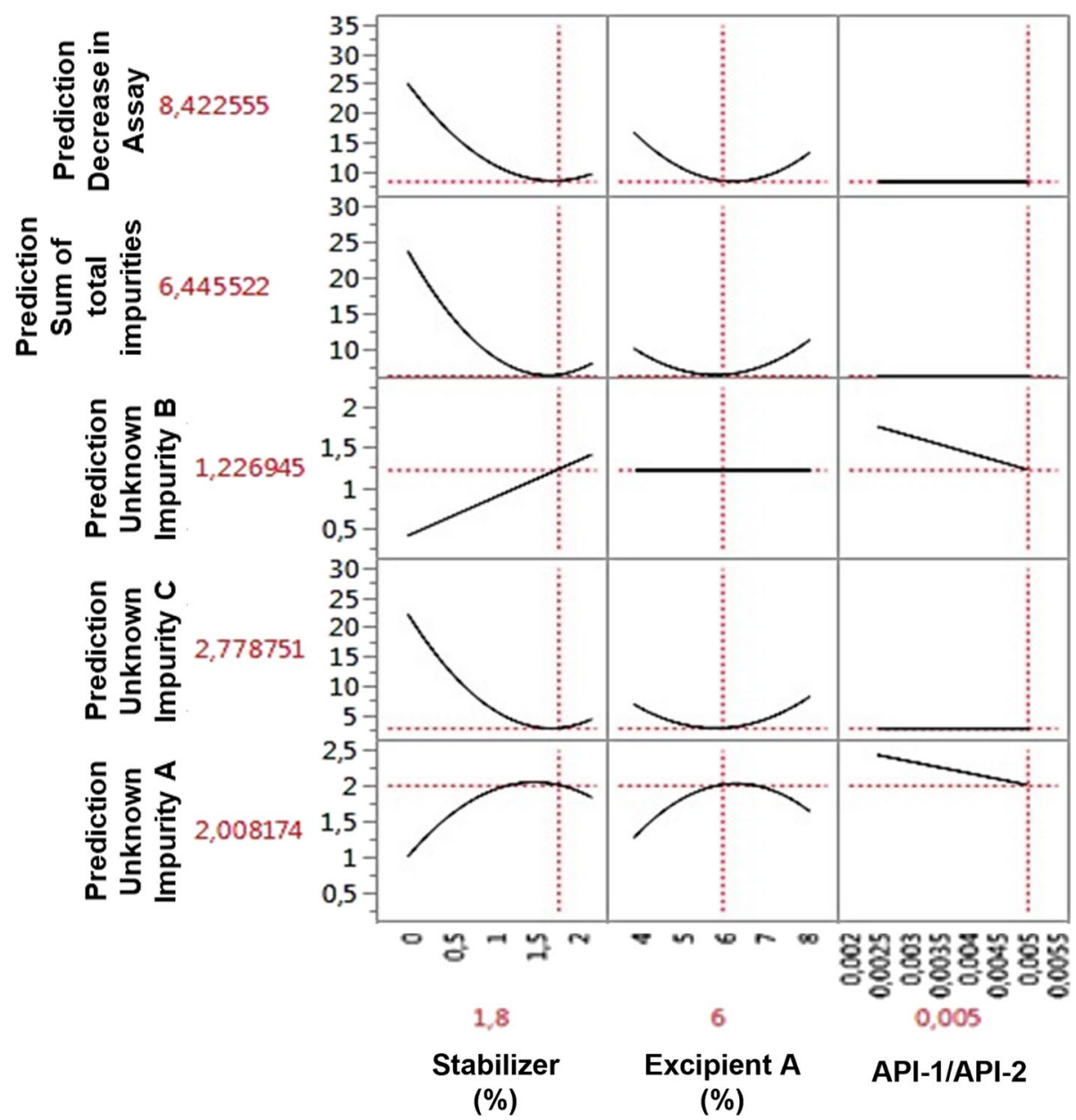

Fig. 3 Optimization of the product formulation using DoE studies. Each plot shows the predicted effect of a given factor in the $x$-axis (formulation component) on each of the responses (y-axis). The red dotted lines indicate the optimized solutions for the responses of interest (lowest impurity levels and lowest decrease in assay in the final drug product)

herein, due to confidentiality reasons). Then, in both cases, the DoE followed a DSD, given the advantages provided by this type of experimental design and the scope of the experimental work (process screening and optimization). Three levels were therefore considered for each factor in both DoEs (low, middle, and high); the DoEs considered 17 runs for UO2 (with 8 PPs used as factors and 7 CQAs as responses) and 13 runs for UO6 (with 6 PPs considered factors and 7 CQAs considered responses). The responses were the same for both DoEs and included relevant quality attributes of the final DP, such as assay, content uniformity, and dissolution. Figure 5 exemplifies how the knowledge obtained from the UO2 DoE analysis can support the identification of CPPs. The right-hand plot shows the true versus predicted values of Assay of API-1 obtained after fitting a multiple linear regression model to the UO2 DoE data. The model constructed to predict Assay of API-1 considers a quadratic term (UO2_PP4:UO2_PP4), three main effects (UO2_PP4, UO2_PP3, and UO2_PP6) and a two-way interaction (UO2_PP3"UO2_PP6). Criticality of UO2_PP3 and UO2_PP4 was thus set to critical (CPPs) in the criticality assessment table of iRISK ${ }^{\mathrm{TM}}$ (left-hand panel in Fig. 5) since the variability of these PPs is directly impacting at least one of the CQAs (assay of API-1 in this case) in a significant way (as given by the calculated $p$-values of the multiple linear models' outcomes; model terms with a $p$-value below 0.05 are considered significant). Parameters UO2_PP1 and UO2_PP2 were also found to be critical, presenting a significant relationship with other CQAs at a 0.05 level (data not shown).

Regarding UO6, parameters UO6_PP1 and UO6_PP3 were found to be critical (data not shown). Besides confirming the criticality of potential CPPs of UO2 and UO6, the DoE results allowed defining a preliminary 


\begin{tabular}{|c|c|c|c|c|c|c|c|c|c|c|c|c|c|}
\hline & & \multicolumn{12}{|c|}{ CQAs } \\
\hline & & Appearance & $\begin{array}{l}\text { Ident. } \\
\text { API-1 }\end{array}$ & $\begin{array}{l}\text { Ident. } \\
\text { API-2 }\end{array}$ & $\begin{array}{l}\text { Assay } \\
\text { API-1 }\end{array}$ & $\begin{array}{l}\text { Assay } \\
\text { API-2 }\end{array}$ & $\begin{array}{c}\text { Content } \\
\text { Uniformity } \\
\mathrm{APl}-1\end{array}$ & $\begin{array}{c}\text { Content } \\
\text { Uniformity } \\
\text { API-2 } \\
\end{array}$ & $\begin{array}{l}\text { Dissolution } \\
\text { APl-1 }\end{array}$ & $\begin{array}{l}\text { Dissolution } \\
\text { API-2 }\end{array}$ & $\begin{array}{c}\text { Degrad. } \\
\text { Products } \\
\text { APl-1 } \\
\end{array}$ & $\begin{array}{c}\text { Degrad. } \\
\text { Products } \\
\text { API-2 } \\
\end{array}$ & $\begin{array}{l}\text { Water } \\
\text { Content }\end{array}$ \\
\hline \multirow{8}{*}{ 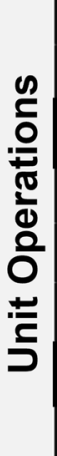 } & U01.1 & Low & Low & Low & MEDIUM & Low & MEDIUM & Low & MEDIUM & Low & HIGH & Low & Low \\
\hline & U01.2 & Low & Low & Low & Low & Low & Low & mediun & Low & mastum & Low & Low & Low \\
\hline & UO2 & HIGH & Low & Low & HIGH & MEDIUM & HIGH & MEDIUM & HIGH & HIGH & MEDIUM & MEDIUM & нІGн \\
\hline & UO3 & MaDUบ & Low & Low & Low & Low & Low & Low & MEDIUM & Magtum & Low & Low & Low \\
\hline & U04.3 & Low & Low & Low & Low & Low & MEDIUM & Low & Low & Low & Low & Low & Low \\
\hline & UO5 & mantum & Low & เow & Low & Low & MEDIUM & Low & НाGH & HIGH & Low & Low & Low \\
\hline & U06 & HIGH & Low & Low & MEDIUM & MEDIUM & HIGH & MEDIUM & HIGH & HIGH & Low & Low & Low \\
\hline & UO7 & HIGH & Low & Low & Low & Low & Low & Low & Mediun & Mabium & mabium & Low & mastum \\
\hline
\end{tabular}

Fig. 4 Criticality assessment of the unit operations (UOs) using iRISK ${ }^{T M}$ Cause-Effect Matrix. The risk of a given UO (in rows) affecting a given product's CQA (in columns) was classified as low (score of 1), medium (score of 3), and high (score of 9). Due to confidentiality reasons, not all of the CQAs are shown. Given their higher overall score, operations UO2 and UO6 (highlighted) were identified as the top 2 UOs potentially affecting the product's Critical Quality Attributes (CQAs)

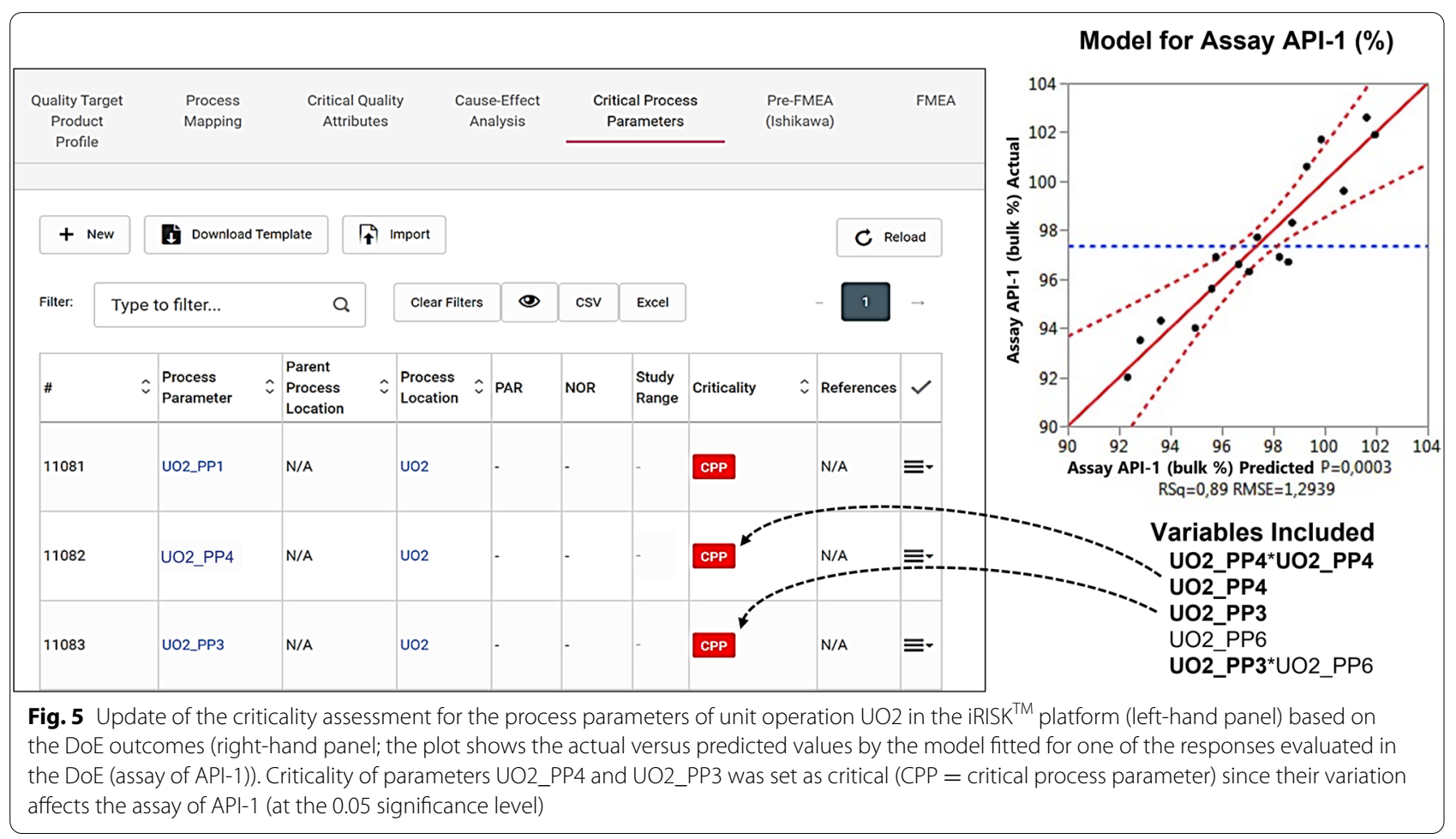

operating range for their PPs to be tested on the scale-up stage. For unit operations without a DoE analysis, results from additional experimental work were used to justify the criticality of their respective PPs. In the absence of evidence to classify a given PP as critical or non-critical, the PP was considered a pCPP. Overall, more than 10
PPs were identified as CPPs in the entire manufacturing process. Since most of the information was obtained at a small scale, the scaling up was a step of utmost importance. A small-scale DS was initially defined considering the knowledge obtained from the $\mathrm{CA}$ and the DoE results. 


\section{Process knowledge, scale-up studies, and control strategy definition}

In the early stages of pharmaceutical process development, investigations are performed at a small scale. Transformations of the small-scale observations into commercial-scale development (Fig. 1) require different design strategies and different equipment which may cause differences in product quality (Raval et al., 2018). To cope with these potential differences in quality due to the presence of scale-up effects when transferring from small scale to commercial scale, the DS in commercial scale must be adapted accordingly.

For the presented case study process, an assessment of the unit operations indicated that both UO2 and UO6 were scale dependent. Ideally, a DoE should be performed at a commercial scale, using the knowledge collected at a small scale as the foundation for selecting PPs to be tested and their respective ranges. This scaleup DoE would allow to (a) confirm the criticality of the PPs, (b) define the optimal ranges for commercial-scale manufacturing, and (c) develop statistical models linking the CPPs with the CQAs. As for this project, it was not feasible to perform a full DoE at a commercial scale for UO2 and UO6. Instead, the process operational ranges for UO2 and UO6 were defined based on a small set of commercial scale batches manufactured at specific conditions, supported by knowledge acquired during the small-scale activities, as described next.

The methodology involved the use of Principal Component Analysis (PCA) and the available production batches (observations): 9 compliant batches (i.e., batches conforming to the acceptance criteria for all CQAs) and one non-compliant batch (i.e., a batch that failed to meet the acceptance criteria for at least one CQA). PCA is a multivariate projection method of data reduction or data compression. It transforms a large set of variables into a smaller dimensional set of new variables designated as principal components (PC), each of which is a linear combination of the original ones. In PCA, the new variables are uncorrelated; the first PC to be extracted (PC1) captures the highest amount of variability in the data set and each successive component accounts for as much of the remaining variability as possible (Jackson, 1991; Esbensen and Geladi, 2009; Næs et al., 2017). The dimensionality reduction provided by PCA allows a simplified representation of the data set, which facilitates exploring and interpreting its correlation structure. This feature of PCA was thus applied at this stage of the project to estimate the process operational ranges for UO2 and UO6. First, a PCA model was built using the values of the selected CPPs for UO2 and UO6 (total of 6 CPPs: 4 for UO2 and 2 for UO6) for the 9 compliant batches. This model allowed to obtain a simplified bidimensional representation of the two major sources of variability of the CPPs for UO2 and UO6, as denoted by the so-called score plot for the first two principal components of the model (PC1 and PC2). The score plot is a scatter plot of the scores of each sample (i.e., the projection of the sample/observation in the PC) on the two components and allows to examine the relationship between samples (Jackson, 1991; Esbensen and Geladi, 2009; Næs et al., 2017). The score plot for PC2 versus PC1 is shown in Fig. 6A, where each green dot corresponds to a compliant batch (total of 9 batches, as mentioned above); these two components capture about $75.4 \%$ of the total variability present in the data. The score plot (Fig. 6A) also shows the predicted scores of the non-compliant production batch (red dot) whose CPPs values were not used to build the original PCA model. The score plot was then used to obtain an initial estimate of the DS for UO2 and UO6: this corresponds to the rectangle area delimited by PC1 and PC2 scores of the compliant batches (green dots), which is outlined by the blue rectangle in Fig. 6A. This region intentionally excludes the predicted non-compliant batch (red dot), since the goal is to define the process operating ranges for $\mathrm{UO} 2$ and $\mathrm{UO} 6$ expected to result in compliance batches. Note that these two components (PC1 and PC2) can be described as a linear combination of the CPPs (not given here), so the selected score plot region can be converted in ranges for each of the 6 considered CPPs of UO2 and UO6.

The next stage of the procedure involved several batch simulation runs, whereby different combinations of the CPPs values within a specified range were randomly chosen to create new hypothetical batches $(100,000$ simulated batches). The first round of 100,000 simulated batches considered a broader range of possible values for UO2 and UO6 CPPs (namely, within 0.75 times below and 1.5 times above the lower and upper limits, respectively, reported by the 10 manufactured batches). The previously derived PCA model was then applied to these simulated batches, and only those batches satisfying the following three criteria were considered "acceptable" batches: (i) predicted score values for PC1 and PC2 within the defined DS estimate (blue rectangle in Fig. 6A); (ii) Hotelling's $T^{2}$ statistics below $80 \%$ of the maximum value obtained by the model, and (iii) a sum of squared residuals below $80 \%$ of the $95 \%$ confidence limit of the model residuals. Hotelling's $T^{2}$ and squared residuals are two useful diagnostic statistics that allow assessing whether a sample has an unusual variance inside the model (sample with large Hotelling's $T^{2}$ ) and/or outside the model (sample with large residuals). Hotelling's $T^{2}$ (or sum of normalized squared scores) measures the distance from a sample to the centre of the model; The sum of squared residuals of a sample provides a measure 

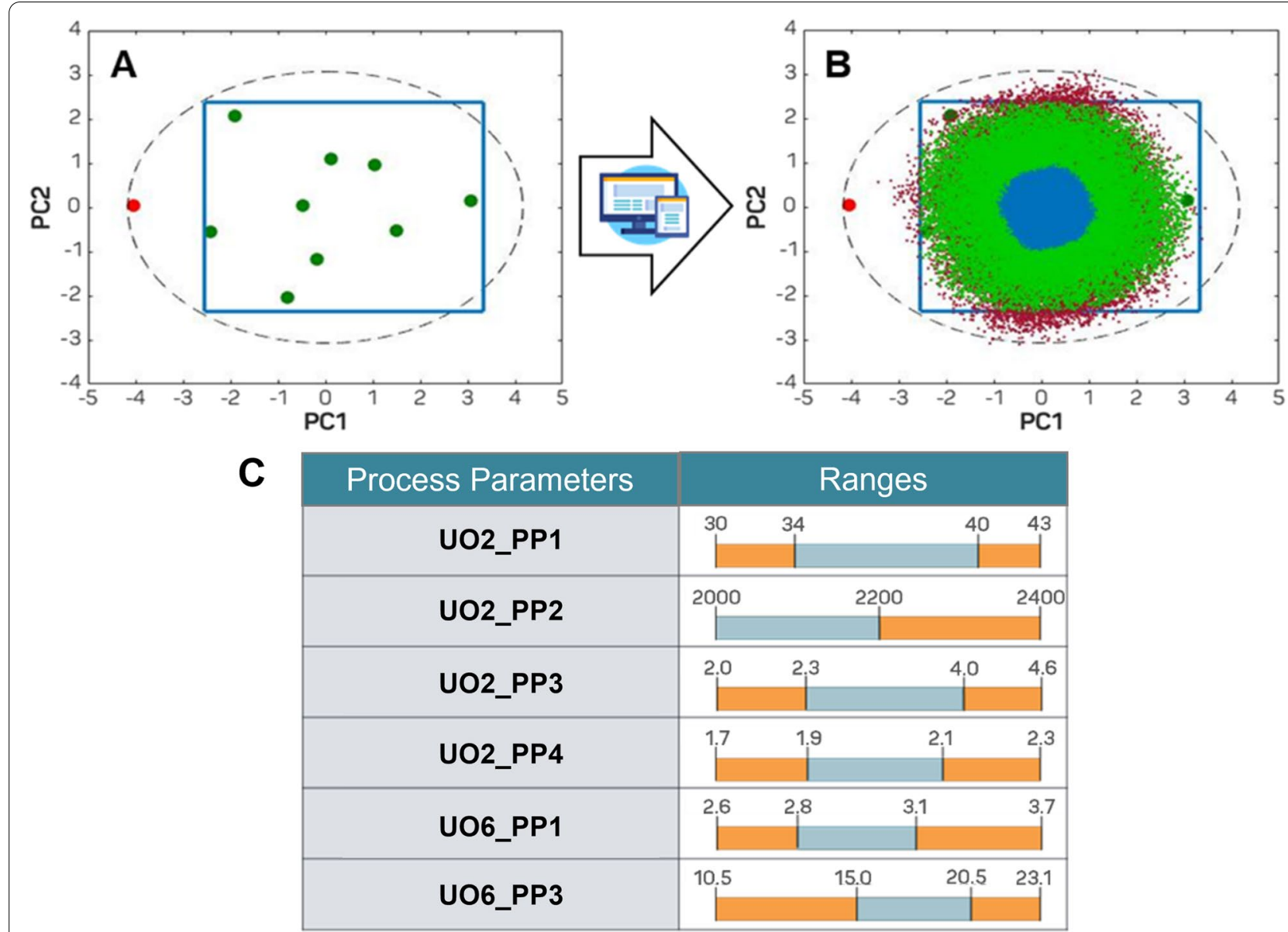

Fig. 6 Definition of the operational ranges for UO2 and UO6 using PCA modelling and computer simulations. Top left panel (A): PCA score plot of the analysis of 9 compliant manufactured batches (green dots). The data set consists of the CPPs values measured for UO2 and UO6; the score plot represents the first two principal components (PC1 and PC2), which describe 53.7\% and 21.7\% of the variance in the data, respectively. The dashed line corresponds to the $95 \%$ confidence ellipse for the model scores. The red dot shows the model predicted scores for a non-compliant production batch. The blue outlined rectangle that covers only compliant batches defines a first estimate of UO2 and UO6 design space (DS). Top right panel (B): PCA model projections for two different sets of batch simulation runs (100,000 samples per run) made to refine the acceptable ranges for $U O 2$ and UO6 CPPs. Each small point corresponds to the predicted model scores for a simulated batch (sample) that has been generated by considering random combinations of the CPPs values within a predefined admissible range (see text for further details). Predicted scores were projected onto the original score plot shown in panel $\mathbf{A}$. The blue outlined rectangle represents the first DS estimate, as defined in panel $\mathbf{A}$, and was used as an acceptance criterion for model predicted scores together with other two diagnostic statistics (Hotelling's $T^{2}$ and sum of squared residuals). The small red and green dots correspond to a set of simulations made after a first refinement of the admissible values for CPPs, where simulated samples satisfying all the acceptance criteria are shown in green. The small blue dots represent the model outcomes for a final set of simulations made after a second (and final) refinement of the CPPs ranges, whereby all simulated batches were found to comply with the predefined acceptance criteria. Bottom panel (C): The table summarizes the initial experimental ranges of the manufactured compliant batches (orange) and the final restricted ranges (blue) obtained for each CPP of UO2 and UO6 based on the described methodology

of the distance between the sample and its projection on the model (i.e., lack of fit of the model to each sample) (Jackson, 1991; Esbensen and Geladi, 2009; Næs et al., 2017). These "acceptable" simulated batches were then employed to perform a first refinement of the CPPs ranges to use for UO2 and UO6, by assuming the 95\% confidence interval for each CPP in the "acceptable" simulated batches. These new ranges of admissible values for CPPs were considered to generate a second set of random batches (100,000 batches). The resulting PCA model predictions are projected on the score plot in Fig. 6B (small red and green dots) and were assessed based on the same acceptance criteria (i)-(iii) outlined above for prediction scores and the two diagnostic statistics. The simulated samples satisfying all the acceptance criteria correspond to the small green points shown in Fig. 6B.

Finally, a second refinement of the allowable ranges for CPPs was made by running consecutive sets of batch 
simulations (100,000 batches per run) within decreasing ranges of CPPs values and assessing their PCA model predictions based on the previously defined acceptance criteria. The widest restricted ranges of CPPs values leading to a $100 \%$ "acceptance" rate of simulated batches were chosen as the final restricted ranges. The model projections for 100,000 simulations computed within these new restricted CPPs ranges are shown by the blue dots in Fig. 6B. The restricted CPPs ranges are disclosed in Fig. 6C (in blue; for comparison, the full ranges for the nine CQA-compliant production batches are included in orange). These CPPs ranges were employed to define the Normal Operating Range (NOR) for UO2 and UO6 and were applied at production to manufacture three validation batches, which fulfilled all quality requirements.

In parallel with the scale-up activities and following the QbD workflow (Fig. 1), a process RA was performed using the Failure Mode and Effect Analysis (FMEA) methodology. With a wide application in manufacturing industries, FMEA is a risk management tool used by many pharmaceutical companies for risk ranking; FMEA provides a systematic method of identifying and preventing system, product, and process problems before they occur (ICH, 2005; ISPE, 2017; ISPE/PDA, 2019; ASQ, 2020; Stamatis, 2003; Stamatis, 2019). Along the FMEA exercise (iRISK, 2021), a multidisciplinary technical team identified, analysed, and prioritized the risks, creating a list of all the failure modes that may occur during commercial manufacturing and the potential effects related to each failure. Additionally, the FMEA allowed the quantification of risks and prioritization for their mitigation and/or elimination by classifying the risk according to the severity of the effect, and the occurrence and detectability probabilities for the failure mode (Fig. 7). The risk priority number (RPN) allows the quantification of risk by multiplying severity, occurrence, and detectability values. Thus, FMEA represents a systematic methodology to rate the risks relative to each other. For that, a rating scale for severity, occurrence, and detectability was agreed between the technical team and applied along the FMEA activity. The scale considered a 5-level rank of even values ranging from 2 to 10 . Additionally, it was defined beforehand a threshold value for RPN (in this case RPN of 288) above which mitigation actions should be defined to reduce the risk.

Severity was attributed according to the impact of the identified risk on the product's quality and compliance, by extension, based on the impact for the patient. For example, if a risk describes an increase outside the operating range for a certain PP and that increase causes the CQA to go out of specification, adversely affecting the patient's health, then the risk severity was classified as very high (rank of 10). If a given failure does not affect the product's quality and the patient's health and safety, its severity is ranked as very low (rank of 2). Likelihood of occurrence was quantified in terms of how often that event might occur during routine batch manufacturing (a rank of 2 if the failure is unlikely; a rank of 4 if the failure has a probability of occurrence below 1\%; a rank of 6 if there are 5 occurrences in 100 events; a rank of 8 if the failure is frequent but with a probability below $10 \%$; and a rank of 10 if the failure is very frequent with a probability of having more than 3 occurrences in 10 events).

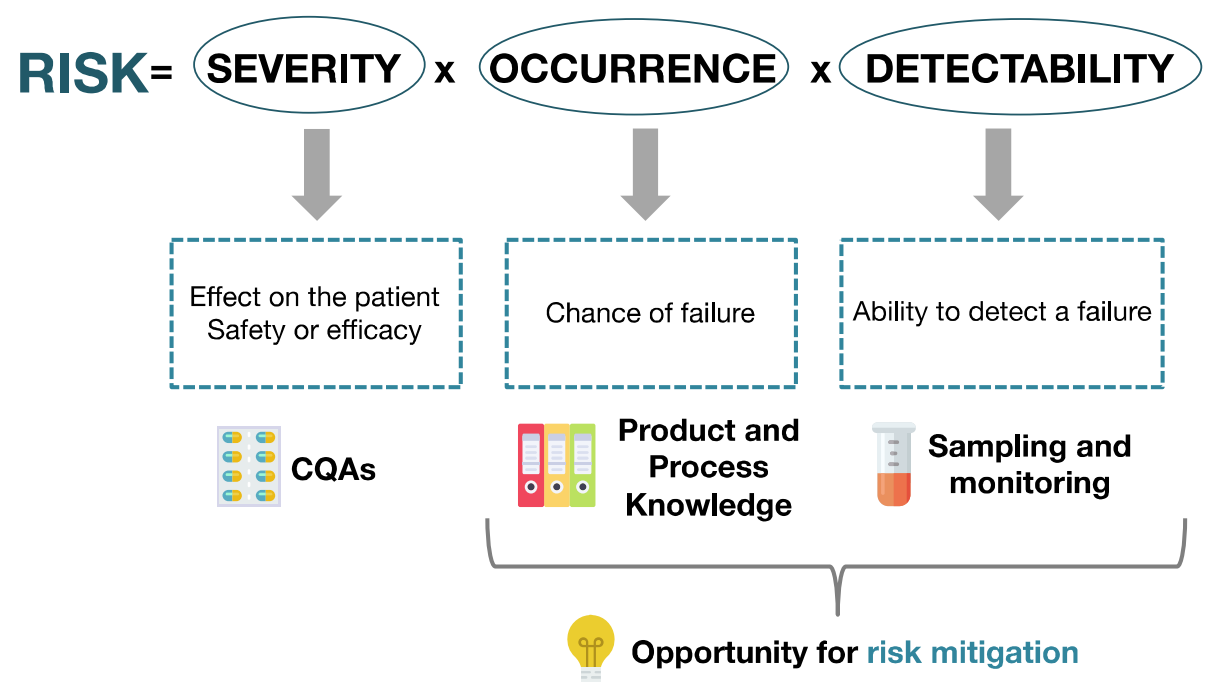

Fig. 7 Decomposition of risk in severity, occurrence and detectability, and identification of the source of knowledge used for their assessment (CQAs, critical quality attributes) 
During scale-up and additional batches manufacture, certain failure modes with a low occurrence frequency during development were often observed (i.e., had high probability of occurrence); this reveals the importance of revising the RA throughout the product's lifecycle. The same principle applies to the likelihood of detection, which quantifies how easy and quick the detection of a certain failure mode is. The detectability scale ranged from 2 (if the failure mode was easily and always detected) to a rank of 10 (when the failure mode was hard to detect and only detected in less than $67 \%$ of the cases).

Along the FMEA, about 100 failure modes were identified, with the majority (90\%) being classified as easily and always detected (detectability rank of 2; and with a RPN not greater than 72) since there were reliable detection controls in place and the process automatically prevented further processing. Moreover, only $10 \%$ of the identified failure modes had a RPN equal to or greater than 128 , but none exceeded the predefined threshold (RPN of 288).

More than $40 \%$ of the failure modes identified in the RA were related to UO2 operation, followed by UO7 operation with about $25 \%$ of the failure modes.

A robust control strategy, with a strong monitoring plan, can help reduce the occurrence and/or improve the detectability of specific failure modes, thus mitigating risk.

After finalizing the RA, despite none of the classified failure modes surpassing the RPN threshold, the technical team decided to address some of the ones with highranking RPN values. For example, a mitigation action was defined for a failure mode with a RPN value of 256 . The action was a verification step for a certain equipment to check for its integrity status. By implementing this mitigation action, the RPN dropped to an acceptable value $(\mathrm{RPN}=128)$ due to an improvement in the failure mode's detectability (detection rank dropped from 8 to a value of 4). This verification step was added to the control strategy as a preventive control.

By the end of process development (Fig. 1), the process control strategy was defined based on the RA exercise and the characteristics of the NOR/DS. The control strategy was formalized in several facilitation sessions with a multidisciplinary team and the support of iRISK $^{\mathrm{TM}}$ risk management platform (iRISK, 2021). The control strategy was composed by preventive controls (e.g., equipment calibration), detective controls (e.g., alarms), and in-process controls, amongst others.

\section{Product lifecycle management}

By adopting QbD during pharmaceutical development, deep process and product understanding were obtained, allowing the creation of a knowledge base for the product. With a higher understanding of the relationship between the process and the product, it is possible to know what impact a certain change in process will have, supporting the decision-making flow (ISPE, 2011). Besides, it is important to update the knowledge base whenever a critical change (e.g., change in supplier, change of equipment) or deviation (e.g., equipment out of calibration, error in following a given operating instruction) occurs. The change control system handles changes done in the context of continuous improvement or by necessity (e.g., change of a raw material supplier). The change must be evaluated with a knowledge and risk-based approach, hence why it is important to keep the risk and knowledge base updated. Depending on the type of change, its implementation might require prior approval from the regulatory authorities (ICH, 2019). There is an interactive flow of information between the risk management and data/knowledge management systems, as represented in Fig. 8. The use of monitoring systems and the establishment of a Continued Process Verification (CPV) plan, as well as the application of data analysis strategies, allow the continuous flow of knowledge regarding the state of the process. This information can be used to update the RA, supporting the identification of new risks that might be detected and revision of existing ones. Depending on their criticality, risks might have to be addressed and the control strategy may need to be improved by implementing risk mitigation actions. An improved control strategy should be able to keep the process in control; this can be monitored in the CPV programme. This flow of information should be managed during the entire product's lifecycle for the resulting knowledge base to be representative of the current situation regarding the product's quality and the process's performance.

Product lifecycle management activities include all that was done through development until the product is no longer commercialized. It is important to look at lifecycle management at a commercial stage through a continuous improvement lens since it is about maximizing the value of the product to the patient (Tiene, 2017). This can include changes in formulation, process unit operations, packaging, delivery systems, or even the inclusion of Information Technology or automation solutions for improving and automatizing the collection and assessment of data and risks. The use of an up-to-date knowledge base regarding the product and process greatly supports the selection of improvement actions since their impact will be better understood.

\section{Conclusion}

This article describes a successful application of Quality by Design to the development of a pharmaceutical generic drug product (coated tablet form). By following a QbD approach, a significant reduction of $30 \%$ in the 


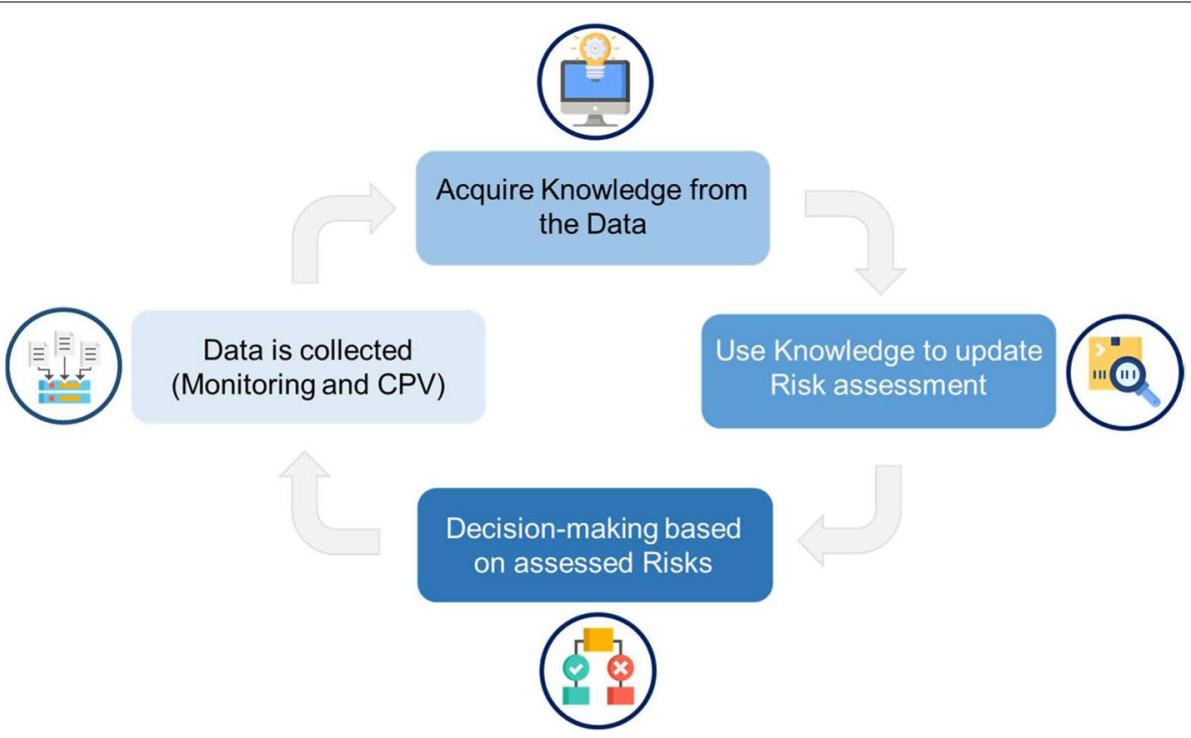

Fig. 8 Flow of data, knowledge, and risk: these are continuously being updated and iterating with each other throughout the product's lifecycle

overall development and validation time was achieved when compared to a traditional approach. The collection of knowledge in a systematic manner allowed the definition of a robust process that will consistently achieve the desired product quality. Future decisionmaking and continuous improvement activities will likewise be supported by the gained product and process understanding. One may expect that its lifecycle management to be much less unpredictable given the much higher level of process and product knowledge established. Additionally, this methodology can be easily transferred to the development of other products, bringing in further acceleration to the standard pharmaceutical development process. Overall, a more efficient and with enhanced quality critical path was followed and shown feasible. This translates into higher quality, safety, and efficacy of medicines for patients.

\section{Abbreviations}

API: Active pharmaceutical ingredient; CA: Criticality assessment; CEM: Causeeffect matrix; CMA: Critical material attribute; CPP: Critical process parameter; CPV: Continued process verification; CQA: Critical quality attribute; DoE: Design of experiments; DSD: Definitive screening design; DP: Drug product; DS: Design space; FMEA: Failure mode and effect analysis; ICH: International Council for Harmonisation of Technical Requirements for Pharmaceuticals for Human Use; NOR: Normal operating range; PCA: Principal component analysis; PC: Principal component; pCMA: Potential critical material attribute; pCPP: Potential critical process parameter; PCQA: Potential critical quality attribute; PP: Process parameter; QbD: Quality by design; QTPP: Quality target product profile; RA: Risk assessment; RM: Raw material; RPN: Risk priority number; UO: Unit operation.

\section{Acknowledgements}

This work was carried out as a collaborative project between 4Tune Engineering and Libbs Farmacêutica. 4Tune Engineering is a consulting company with over 18 years of experience in the pharmaceutical area. Founded in 1958, Libbs Farmacêutica is a pharmaceutical company in the forefront of key innovation projects within Brazil's industry: it is a pioneer in the launch of biosimilars and monoclonal antibodies in Brazil and currently produces ninety different products. We would like to thank all the departments involved in the project at Libbs Farmacêutica for their support, commitment, and dedication; we would also like to thank João Almeida Lopes for his technical contribution.

\section{Authors' contributions}

All authors contributed to the study's conception and design. Material preparation, data collection, and analysis were performed by MT, TCS, and LPM. The first draft of the manuscript was written by MT and critically reviewed, commented, and edited by LPB. All authors commented on previous versions of the manuscript. The final manuscript was prepared by MT and LPB. All authors read and approved the final manuscript.

\section{Availability of data and materials}

The datasets generated during and/or analysed during the current study are not publicly available due to confidentiality reasons.

\section{Declarations}

Ethics approval and consent to participate

Not applicable.

\section{Consent for publication}

Not applicable.

\section{Competing interests}

The authors declare that there is no conflict of interest involved in the preparation of this article.

\section{Author details}

${ }^{1}$ 4Tune Engineering Lda., Av. António Augusto Aguiar 108-4, 1050-019 Lisbon, Portugal. ${ }^{2}$ Department of Research and Development, Libbs Farmacêutica Ltda, Embu das Artes, SP, Brazil. ${ }^{3}$ 4Tune Engineering Lda., Brazil Office, Avenida Vereador Narciso Yague Guimarães, 1145; Sala 1008 - Helbor Concept Office, 
São Paulo, Brazil. ${ }^{4}$ Department of Production, Libbs Farmacêutica Ltda, Embu das Artes, SP, Brazil.

Received: 14 May 2021 Accepted: 6 December 2021

Published online: 24 December 2021

\section{References}

American Society for Quality (ASQ). Failure Mode and Effects Analysis (FMEA) https://asq.org/quality-resources/fmea. Accessed October 2020

Esbensen KH, Geladi P (2009) 2.13 - Principal component analysis: concept, geometrical interpretation, mathematical background, algorithms, history, practice. In: Brown SD, Tauler R, Walczak B (eds) Comprehensive chemometrics. Elsevier, Oxford, p 211-226. ISBN 9780444527011 . https:// doi.org/10.1016/B978-044452701-1.00043-0.

International Conference on Harmonisation of Technical Requirements for Registration of Pharmaceuticals for Human Use (ICH) (2005) ICH Harmonised Tripartite Guideline - Q9 Quality Risk Management. https://datab ase.ich.org/sites/default/files/Q9\%20Guideline.pdf.

International Conference on Harmonisation of Technical Requirements for Registration of Pharmaceuticals for Human Use (ICH) (2008) ICH Harmonised Tripartite Guideline - Q10 Pharmaceutical Quality System. https:// database.ich.org/sites/default/files/Q10\%20Guideline.pdf.

International Conference on Harmonisation of Technical Requirements for Registration of Pharmaceuticals for Human Use (ICH) (2009) ICH Harmonised Tripartite Guideline - Q8(R2) Pharmaceutical Development. https:// database.ich.org/sites/default/files/Q8\%28R2\%29\%20Guideline.pdf.

International Council for Harmonisation of Technical Requirements for Registration of Pharmaceuticals for Human Use (ICH) (2019) ICH Harmonised Guideline - Q12 Technical and Regulatory Considerations for Pharmaceutical Product Lifecycle Management. https://database.ich.org/sites/defau It/files/Q12_Guideline_Step4_2019_1119.pdf.

International Society for Pharmaceutical Engineering (ISPE) (2011) Part 1 - Product Realization using Quality by Design (QbD): Concepts and Principles. In: ISPE Guide Series: Product Quality Lifecycle Implementation $\left(P Q I^{\circledR}\right)$ from Concept to Continual Improvement. www.ispe.org.

International Society for Pharmaceutical Engineering (ISPE) (2017) Volume 7 - Risk-based manufacture of pharmaceutical products. 2nd ed. In: ISPE Baseline ${ }^{\circledR}$ Guide. www.ispe.org.

International Society for Pharmaceutical Engineering (ISPE) / Parenteral Drug Association (PDA) (2019) ISPE - PDA guide to improving quality culture in pharmaceutical manufacturing facilities. https://www.ispe.org/sites/ default/files/regulatory/ispe-pda-guide-to-improving-quality-culture.pdf.

iRISK ${ }^{\mathrm{TM}}$. The next generation risk management platform, https://www.risk. com. Accessed Feb 2021.

Jackson JE (1991) A user's guide to principal components, 1st edn. John Wiley \& Sons, New York. ISBN 0-471-62267-2.

Malik A, Gochhayat G, Alam M, Kumar M, Pal P, Singh R, Saini V (2019) Quality by design: a new practice for production of pharmaceutical products. J Drug Deliv Ther 9(1-S):416-424:10.22270/jddt.v9i1-s.2370

Montgomery DC (2020) Design and analysis of experiments. 10th edn. John Wiley \& Sons, New York. ISBN 978-1119722106.

Næs T, Isaksson T, Fearn T, Davies T (2017) A user-friendly guide to multivariate calibration and classification, 2nd edn. NIR Publications, Chichester. ISBN 978-1-906715-25-0.

Raval N, Tambe V, Maheshwari R, Deb PK, Tekade RK (2018) Chapter 19 Scale-up studies in pharmaceutical products development. In: Tekade RK (ed) Advances in Pharmaceutical product development and research, dosage form design considerations. Academic Press, p 669-670. ISBN 9780128144237. https://doi.org/10.1016/B978-0-12-814423-7.00019-8.

SAS Institute, Inc. (2019) JMP ${ }^{\circledR} 15$ Design of experiments guide. SAS Institute Inc., Cary, NC.

Stamatis DH (2003) Failure Mode and Effect Analysis: FMEA from theory to execution, 2nd edn. https://asq.org. American Society for Quality (ASQ) Quality Press. ISBN 978-0-87389-598-9.

Stamatis DH (2019) Risk Management Using Failure Mode And Effect Analysis (FMEA). https://asq.org. American Society for Quality (ASQ) Quality Press. ISBN 978-0-87389-978-9.

Tiene G (2017) Lifecycle management strategies can uncover hidden value. Pharma Manufacturing (internet; cited 8 Mar 2017). Available via https:// www.pharmamanufacturing.com/articles/2017/lifecycle-managementstrategies-can-uncover-hidden-value/.

Yu LX, Amidon G, Khan MA, Hoag SW, Polli J, Raju GK, Woodcock J (2014) Understanding pharmaceutical quality by design. AAPS J 16(4):771-783. https://doi.org/10.1208/s12248-014-9598-3

\section{Publisher's Note}

Springer Nature remains neutral with regard to jurisdictional claims in published maps and institutional affiliations.

\section{Submit your manuscript to a SpringerOpen ${ }^{\circ}$ journal and benefit from:}

- Convenient online submission

- Rigorous peer review

- Open access: articles freely available online

- High visibility within the field

- Retaining the copyright to your article

Submit your next manuscript at $\boldsymbol{\nabla}$ springeropen.com 